\title{
Ausstellen, Forschen, Lehren
}

\section{Das medizinhistorische Museum zwischen universitärer Medizin und Öffentlichkeit}

\author{
Thomas Schnalke
}

Die Medizin steuert von jeher einen gewichtigen Teil zur universitären Sammlungskultur bei, deren Stand Anke te Heesen in ihrem Beitrag zum Forum 4/2008 umrissen hat. In den verschiedenen Einrichtungen ihrer Fakultäten - Abteilungen, Institute, Kliniken - finden sich einschlägige, vom Einsatz in der aktuellen Forschung und Lehre, Diagnostik und Therapie freigestellte Objekte. Diese lagern vereinzelt und teilweise übersehen in zugigen Fluren und dunklen Kellern, bisweilen reüssieren sie in kleineren und größeren Vitrinen auf den Gängen vor Hörsälen oder Sekretariaten. Hin und wieder sind sie aber auch eingestellt in ausgewiesene Sammlungsräume. Mancherorts wird damit schließlich gar versucht, wie derzeit etwa im Hamburger Universitätsklinikum Eppendorf, ein eigenes Museum aufzubauen.

Vollgültige medizinische beziehungsweise medizinhistorische Museen als Gliederungen einer medizinischen Fakultät gibt es im deutschen Sprachraum allerdings nur in Zürich und Wien, Bochum und Berlin. Im Spannungsfeld zwischen universitärer Einbettung und öffentlicher Wirkung übernehmen sie spezifische Rollen und Funktionen und bieten den beteiligten Akteuren bestimmte Möglichkeiten. Meine eingehendere Auslotung dieses 
Terrains am Beispiel des Berliner Medizinhistorischen Museums der Charité soll zur Diskussion über die gegenwärtige und künftige Bedeutung derartiger Einrichtungen beitragen. Im Sinne te Heesens verstehe ich diese Ausführungen als Plädoyer für eine verstärkt zu betreibende materiale Kultur in der Medizin- und Wissenschaftsgeschichte und damit als ein positiv gestimmtes Gegengewicht zur „pessimistischen Zuspitzung“, wie es Christian Sichau in seinem Beitrag zum Forum in Heft 1/2009 mit Blick auf technikhistorische Museen formuliert hat. Durchaus zutreffend hat er darin auf eine verbreitet anzutreffende museale Starre verwiesen, die sich vor dem Hintergrund voller Depots und einer unübersehbar großen Flut sperriger Black-Box-Geräte sowie wissenschaftlicher Praktiken, die sich im Unsichtbaren verlieren, in einer rein auf die unmittelbare Ausstellungspraxis reduzierten Sammlungsaktivität erschöpft.

Eine nähere Standortbestimmung jeglicher musealer Aktivität beginnt sinnvollerweise mit einer genaueren Betrachtung des Umfelds, in das die betreffende Institution eingebettet ist. Als Teil der universitären Medizin liegt das Berliner Medizinhistorische Museum auf dem historischen Campus der Charité in der Mitte der Stadt. Es ist somit integraler Bestandteil eines akademischmedizinischen Komplexes, der klassischerweise durch die Trias Forschung, Lehre und Krankenversorgung ausgewiesen ist und der überdies seine eigene vielschichtige und zeitweise als hochbedeutsam angesehene Vergangenheit besitzt. Diese Topographie belegt das Museum, das ebenfalls seine eigene, über einhundertjährige Geschichte hat, von vornherein mit etlichen Ansprüchen, welche die Akteure in dieser Einrichtung geflissentlich ignorieren oder synergetisch integrieren können. So wirkt das allfällige medizinisch-universitäre Moment unweigerlich in das Museum hinein und potenziell durch dieses hindurch in die interessierte Öffentlichkeit hinaus. Im gegenläufigen Sinne registriert das Museum aber auch Impulse aus der Öffentlichkeit, die nach innen in die Fakultät weitergeleitet werden können, so dass das Museum gerade mit seinem öffentlichen Auftritt auf die universitäre Medizin zurückwirkt.

Der bewusste Kurzschluss mit Akteuren der aktuellen Medizin bietet die Chance, einen vielschichtigen Dialog zu etablieren und daraus Fragestellungen zu entwickeln, die sich im Museum in ihren historischen und aktuellen Bezügen vor die Öffentlichkeit bringen lassen. Die gegenwärtige Medizin ist zudem eine eminente Quelle ausstellungswürdiger Objekte und hält überdies Expertisen 
bereit für die Identifikation und nähere Funktionsbestimmung rezenter wie auch älterer Gegenstände. Schließlich besteht die Möglichkeit, für einzelne Ausstellungsprojekte gezielte Kooperationen mit Mitarbeiterinnen und Mitarbeitern aus medizinischen Einrichtungen im Umfeld vor Ort anzubahnen und einschlägige Projekte gemeinsam zu realisieren. Dies wurde im Berliner Medizinhistorischen Museum kürzlich exemplarisch mit der Ausstellung „Vom Tatort ins Labor“ versucht, die in enger Kooperation zwischen Verantwortlichen des Museums und des Instituts für Rechtsmedizin der Charité entstand.

Nach außen gewinnt ein medizinhistorisches Museum, wie jede Einrichtung seiner Art, durch seine Ausstellungen Profil, die auf bestimmte Erwartungshaltungen in der Öffentlichkeit treffen. Bei diesen externen Vorannahmen handelt es sich um Zuschreibungen seitens der Museumsklientel, die sich in unserem Fall grob in zwei Gruppen trennen lässt: das wissenschaftliche Publikum aus den verschiedenen Sparten der medizinischen Fachwelt und die im besten Falle fachlich interessierten, aber nicht einschlägig medizinisch vorgebildeten Laien. Auch wenn sich viele Besucherinnen und Besucher dem Museum als einer Einrichtung der Medizin nähern, in der sie Erläuterungen und Verlautbarungen aus diesem Gebiet erwarten, bietet sich für die Akteure des Museums geradezu eine gegensätzliche Grundhaltung an. Um ein Publikum jenseits der vielfach als hermetisch empfundenen Eingrenzungen des medizinischen Komplexes zu erreichen, empfiehlt es sich, den Wirkraum des Museums als eine Sphäre der allgemeinen Öffentlichkeit zu definieren. Hier ist die Medizin zu Gast. Sie nutzt die öffentlich legitimierte Bühne, um sich aus ihrer spezifischen Geschichte und ihren aktuellen Bezügen heraus zu erklären.

Thematisch gebundenes Ausstellen $\mathrm{zu}$ medizinischen und medizinhistorischen Themen meint gerade in einem universitären Umfeld ein wissenschaftlich gegründetes Veröffentlichen - anders gesagt, die Ausstellung ist in diesem Zusammenhang ein Publikationsformat eigener Qualität. Im Unterschied zu diskursiven, durch Fußnoten, Literaturlisten und Anhänge belegten Textbeiträgen, die ihre Argumentationsstruktur auf einer endlichen Seitenzahl über das geschriebene Wort aufbauen, entwickeln die Erkenntnisgebäude einschlägiger Ausstellungen ihre inhaltlichen Aussagen primär über Objekte, die durch erläuternde und kommentierende Texte in eine verräumlichte Syntax der Dinge mit vorgegebener Quadrat- oder Kubikmeterzahl eingefügt und somit 
in eine anschauliche, spannungsreiche und aussagekräftige Beziehung zueinander gestellt sind. Diese Verräumlichung des Wissens, dieses Inbeziehungsetzen hinreichend entschlüsselter Realien liefert die Grundmodule einer szenischen Darbietung des medizinund wissenschaftshistorischen Gedankengangs im Sinne einer weitgezogenen Kontextualisierung, die bestenfalls Elemente einer diskursiven Analyse mit einer dramatischen Erzäh-lung vereint. Über die räumliche Kopplung von derartig zusammengehörigen und gleichzeitig voneinander abgrenzbaren, sich gegenseitig ankündigenden, eingrenzenden und in Frage stellenden Ausstellungsgedanken in sorgfältig ausgewählten und arrangierten Objektgruppen wird die Argumentationsstruktur und damit letztlich auch der Spannungsbogen einer dreidimensionalen Veröffentlichung im Museum aufgebaut.

Wie jeder Text, so will sich auch jede Ausstellung bei ihrem Publikum verständlich machen. Hierzu verfügt das medizinhistorische Museum über eine Reihe spezifischer Mittel, die diese Einrichtung zu einem Forum für die Begegnung zwischen medizinischer Fachwelt und Öffentlichkeit machen. Mit seinen aus der Geschichte abgeleiteten Positionen bietet es über die Vorstellung, Erklärung und Kommentierung medizinischer, gesundheits- und krankheitsrelevanter Sachverhalte nicht nur Aufklärung, Information und bis zu einem gewissen Grade Unterhaltung, sondern auch ein Stück weit Erleichterung und Distanz. Bei allen möglichen Spiegeleffekten kommen der Besucher und die Besucherin (hoffentlich) ins Museum als gesunde Gäste, die möglicherweise zwar eine potenzielle „Kränkbarkeit“ des eigenen Körpers, eine grundsätzliche Gefährdung des eigenen Selbst wahrnehmen, diese aber auch immer über das Erkennen der momentanen Gesundheit rationalisieren und damit produktiv aufheben können.

Aus der Möglichkeit zur Distanz erwächst im medizinhistorischen Museum eine unter den Besuchern häufig anzutreffende aufmerksame Gelassenheit, welche beide Bereiche - Medizin und Gesellschaft - produktiv zu verschränken mag. Daraus speist sich nicht selten ein konstruktiver Dialog, der heute angesichts der Entwicklungen in der Medizin und der sich daran anknüpfenden theoretischen, ethischen und philosophischen Debatten wichtig erscheint. An dieser Stelle kann das medizinhistorische Museum zusätzlich zu seiner Funktion als Publikationsforum den Part eines Moderators übernehmen.

Das Ausstellungswesen des Berliner Medizinhistorischen Museums gliedert sich in einen Sonder- und einen 
Dauerausstellungsbereich. Seine temporären Präsentationen thematisieren Medizinisches und Medizinhistorisches bewusst breit gehalten in vielfältigen Kontexten, so etwa in wissenschaftlichen, kulturellen, gesellschaftlichen, religiösen und politischen Bezügen. Einzige Vorgabe ist, dass in allen Wechselausstellungen stets ein deutlicher medizinischer Kern sichtbar werden muss. Das historische Moment ist den einschlägigen Konzeptionen vielfach inhärent, kann aber auch bisweilen für das eine oder andere Projekt bewusst kurz gehalten werden oder ganz entfallen. Mit der zeitweiligen Konzentration auf das Gegenwärtige mag - worauf Sichau eindringlich hinweist - die Gefahr bestehen, dass die Schleusen für eine Ausstellungskultur geöffnet werden, die den geschichtlichen Aspekt den Werbestrategien der Sponsoren opfert. Allerdings gehört es bislang nach wie vor zu den professionellen Grundaufgaben seriösen Ausstellungsmachens, den redaktionellen Teil deutlich vom Werbe-block zu trennen, ohne den freilich heute - prominent oder dezent inszeniert - keine größere Ausstellung mehr auskommt.

Bei Themenwahl und -umsetzung in einer medizin- wie wissenschaftshistorischen Ausstellung auf gegenwärtige Bezüge gänzlich verzichten $\mathrm{zu}$ wollen, ist weder ratsam noch möglich. Zum einen reflektiert jede Form der Veröffentlichung, somit auch jene in den Schauräumen eines Museums, bis zu einem gewissen Grad auch die in der jeweiligen Aktualität angekommene Biographie des Veröffentlichenden und durch diesen hindurch die Gefasstheit einer Kultur in der Gesellschaft zur Zeit der Realisierung des Ausstellungsprojekts. Zum anderen ergibt sich gerade im Museum für die Besucherinnen und Besucher einschlägiger Ausstellungen die gar nicht triviale Frage: Was hat das hier Gezeigte mit mir zu tun?

Nach innen, als Teil einer medizinischen Fakultät, bemüht sich das Berliner Medizinhistorische Museum mit seinen Sammlungsbeständen um spezifische Ansätze in Forschung und Lehre. Dabei ist zwar, wie Anke te Heesen für den Bereich universitärer Sammlungen allgemein konstatiert, auch für diesen Ort eine Diskrepanz zwischen einer verbreiteten Rhetorik, die auf eine Intensivierung des materialen Ansatzes in der Wissensforschung und Wissenschaftsgeschichte abhebt, und einer Zurückhaltung der Forscherinnen und Forscher festzustellen, sich tatsächlich in die einschlägigen Sammlungen zu begeben, um ihre Studien mit den vorfindbaren dinglichen Quellen durchzuführen. Zudem lässt sich diese Enthaltsamkeit auch für den Bereich der universitären Lehre feststellen, in der trotz vieler innovativer Ansätze bis heute erstaunlich beharrlich die eingeübten Rituale im white cube des 
klassischen Seminarraums dominieren. Jedoch spricht all dies nicht gegen eine intensivere Einbeziehung medizin- und wissenschaftsgeschichtlichen Sammlungsguts in Forschung und Lehre. Vielmehr zeigt sich hier als ein eminentes Desiderat nur die unbedingte Notwendigkeit, Räume und Formate $\mathrm{zu}$ entwickeln, die eine intensivere Beschäftigung mit Sachzeugen erleichtern und attraktiv machen. Bezogen auf die Forschung lässt sich feststellen, dass es mit Blick auf die Medizingeschichte im deutschen Sprachraum keine Orte gibt, an welchen - etwa analog zu wohlsortierten und -organisierten Handschriftenabteilungen - Objekte in benutzerfreundlicher Weise ,,ausgehoben“ und vorgelegt werden. Zu fordern wäre somit die Konzeption und Umsetzung einer Art Objektlabor, das die relevanten Dinge unter Wahrung aller konservatorischen Erfordernisse für einen fortgesetzten Abgleich mit anderen Quellen einschließlich der am gleichen Ort verfügbaren Sekundärliteratur bereitstellt.

Auf dem Terrain der Lehre bedient das medizinhistorische Museum zunächst durchaus klassische Unterrichtsformen Vorlesung, Kurs, Seminar -, allerdings geschieht dies vorzugsweise aus dem Museum heraus und unter Einbindung der vorfindbaren Objektwelten. In diesem Sinne lässt sich die Einrichtung mit ihren spezifischen Depots und Schauflächen als eine Art medizingeschichtliches Lehrkabinett verstehen und nutzen, in dessen „dichten“ Räumen - analog zu dichten Beschreibungen eine anschauliche und zugleich differenzierte Auseinandersetzung mit historischen, ethischen und theoretischen Sachverhalten angestoßen werden kann. Darüber hinaus bietet das Museum aber auch die Chance, neue Lehrformen zu entwickeln und zu erproben. So finden im Berliner Medizinhistorischen Museum einerseits etwa in Kooperation mit Dozentinnen und Dozenten vorklinischer und klinischer Fächer vernetzte Unterrichtsveranstaltungen statt, in denen, angeregt durch einzelne ausgewählte, in besonderer Weise auf den gesunden oder kranken Körper verweisende historische Objekte, aktuelle wie auch geschichtliche Lehrinhalte vermittelt werden. Andererseits eröffnet sich im Museum aber auch die Möglichkeit, dass Studierende zu Akteuren werden. Sie können die Schauräume nutzen, um selbstgewählte Themen in Referaten, Führungen oder Postern ihren Kommilitonen, anderen Angehörigen der medizinischen oder akademischen scientific communities oder auch der allgemeinen Öffentlichkeit zu präsentieren. Schließlich lassen sich auch 
Projektseminare mit dem Ziel anbieten, eigene Ausstellungen zu konzipieren und umzusetzen.

Die Erfahrung zeigt, dass die Enträtselung medizinischer und medizinhistorischer Objekte, also die Rekonstruktion der eingeschriebenen geschichtlichen und aktuellen Funktions- und Bedeutungszusammenhänge, aber auch das nachgeschaltete argumentierende Inbeziehungsetzen der bearbeiteten Stücke im Ausstellungsraum einen besonderen Lerneffekt hat. Oftmals entzünden sich erst an der Abfassung und Diskussion von Ausstellungstexten zu primär häufig textlosen Stücken zentrale Fragen, die mit Blick auf die öffentlich zu verantwortende Präsentation nach schlüssigen und redlichen Antworten verlangen. Gerade an diesem Punkt wird die wichtige Vermittlungsfähigkeit und damit letztlich auch jenes Instrument geschult, mit welchem jede Ärztin und jeder Arzt dem Patienten komplexe medizinische Sachverhalte erklären muss.

Thomas Schnalke

Berliner Medizinhistorisches Museum der Charité

Charitéplatz 1

D-10117 Berlin

Deutschland

E-Mail: thomas.schnalke@charite.de 